\title{
CALCULATION OF SPECTROSCOPIC AMPLITUDES USING BOSON EXPANSION WAVE FUNCTIONS
}

\author{
D. BRAUNSCHWEIG \\ Cyclotron Laboratory, Department of Physics, The University of Michigan, \\ Ann Arbor, Michigan 48105 ${ }^{\dagger}$
}

Received 1 May 1974

(Revised 4 November 1974)

\begin{abstract}
A formalism is derived which allows the evaluation of spectroscopic amplitudes for twonucleon transfer reactions. The transfer is assumed to take place between low-lying collective states described by boson expansion wave functions. Numerical calculations have been performed for $(t, p)$ reactions on ${ }^{148,150,152} \mathrm{Sm}$. Excellent agreement is obtained for cross sections leading to the ground state in the residual nucleus.
\end{abstract}

\section{Introduction}

It is well known that two-nucleon transfer reactions can provide a rigorous testing ground for nuclear wave functions ${ }^{1}$ ). This is due mainly to the critical dependence of the cross section on the coherent interference of terms with different principal quantum number for the c.m. motion of the transferred nucleons. Since these terms are proportional to the nuclear overlap coefficients, $\beta_{\gamma L S J}$, we may test the goodness of the wave functions obtained from a nuclear structure model by evaluating these coefficients $\beta_{\gamma L S J}$. In two-nucleon transfer reactions, in contrast to the simpler one-nucleon transfer processes, we cannot extract a spectroscopic amplitude from the experimental cross section due to the interference mentioned above. In order to evaluate the cross section, it is therefore essential to be able to calculate the $\beta_{\gamma L S J}$ starting from a nuclear structure model. For low-lying collective states, it has in general been possible to predict these spectroscopic amplitudes only in the limiting cases where the relevant nuclei are either spherical ${ }^{2}$ ) or well deformed ${ }^{3}$ ), while for nuclei in the transition region no satisfactory formalism has been available.

It is perhaps not so surprising then, that in the last decade or so, considerable effort has been expended in obtaining a unified theory of collective motion. We believe that one of the most successful approaches in this respect is provided by the boson expansion method. This technique was first introduced by Belyaev and Zelevinsky ${ }^{4}$ ) who used it to understand anharmonic effects in vibrational nuclei. The basic idea is to express a microscopic Hamiltonian in terms of boson operators. This is done by

† Supported by the US National Science Foundation. 
expanding the fermion pair and one-body operators in the Hamiltonian in terms of boson operators, in such a way as to preserve the appropriate commutation relation for the fermion pair operators. S $\phi$ rensen ${ }^{5}$ ) extended the scope of the boson expansion method by performing calculations within the transition and deformed regions. In doing so, Sфrensen had to introduce a corrclated boson by performing a canonical transformation within the boson space. Later Kishimoto and Tamura ${ }^{6}$ ) introduced sixth-order corrections to the boson Hamiltonian which were shown to be important in the deformed limit. The rather impressive results ${ }^{7}$ ) obtained for the quadrupole moments and electromagnetic transition strengths tend to indicate that the wave functions obtained with the boson expansion method do indeed offer a good description for low-lying collective states.

In this paper we intend to check the boson expansion wave functions further by calculating the spectroscopic amplitudes for two-nucleon transfer reactions. It should be noted that these spectroscopic amplitudes are formally similar to the electromagnetic transition matrix elements. Therefore we are particularly interested in studying two-nucleon transfers between nuclei which have a markedly different deformation. We expect the two-nucleon transfer amplitudes between such nuclei to be a more sensitive probe of the nuclear wave functions than the electromagnetic matrix elements. In pursuing this program we would then have developed a technique which will give us spectroscopic amplitudes with equal ease if either target or residual nucleus is spherical or deformed. Furthermore, low-lying collective states are amenable to strong inelastic excitations in both incoming and outgoing channels. Therefore, our approach will be general enough so that the spectroscopic amplitude connecting any pair of low-lying collective states in the target and residual nucleus can be calculated with equal ease.

In sect. 2 we give a brief account of the formulation which was developed for this purpose. In contrast to the Hamiltonian, the two-particle transfer operator is nonhermitian and is in general a spherical tensor of rank greater than zero so that some extensions are required of the techniques introduced in the works referred to above. Whenever possible we follow the notation of ref. ${ }^{6}$. In sect. 3 we apply the results of sect. 2 to the study of $(t, p)$ reactions on the isotopes ${ }^{148,150,152} \mathrm{Sm}$. We have chosen these $\mathrm{Sm}$ isotopes since they show a gradual transition from spherical $\left({ }^{148} \mathrm{Sm}\right)$ to well deformed $\left({ }^{152} \mathrm{Sm}\right)$.

\section{Formulation}

\subsection{THE SPECTROSCOPIC AMPLITUDE}

The purpose of this section will be twofold. First, we describe the basic steps involved in obtaining the spectroscopic amplitude with the boson expansion method, and secondly, we will discuss the approximations which have been introduced. 
We define the two-nucleon spectroscopic amplitude $\beta$ by $^{\dagger}$ :

$$
\beta_{\gamma L S J T}\left(J_{\mathrm{i}}, J_{\mathrm{f}}\right)=\left\langle\Psi_{J_{\mathrm{r}}}(A+2) \mid\left[\phi_{\gamma L S J T}\left(\boldsymbol{r}_{1}, \boldsymbol{r}_{2}\right) \psi_{J_{\mathrm{i}}}(A)\right]_{J_{\mathrm{r}}}\right\rangle .
$$

The notation $\left[A_{J_{1}} B_{J_{2}}\right]_{J_{3} M_{3}}$ will be used throughout to denote vector coupling. The quantity $\phi_{\gamma L S J T}\left(r_{1}, r_{2}\right)$ represents the wave function for the two transferred particles. Since the expansion of this factor in terms of boson operators is central to the evaluation of (1), we will discuss the approximations needed at length in the next subsection. The $\psi_{J_{i}}(A)$ stands for the wave function of a target nucleus of $A$ nucleons, which is specified by a complete set of quantum numbers $J_{i}$. The wave function of the residual nucleus $\psi_{J_{\mathrm{r}}}(A+2)$ should be understood in a similar fashion.

The wave functions $\psi_{J_{1}}(A)$ and $\psi_{J_{\mathrm{r}}}(A+2)$ are calculated by making use of the boson expansion method $\left.{ }^{7}\right)$. We may therefore write them as:

$$
\begin{gathered}
\psi_{J_{2} M_{\mathrm{i}}}(A)=\sum_{N v \eta} B_{N v \eta^{\prime}}^{J_{2}}\left|N v \eta J_{\mathrm{i}} M_{\mathrm{i}}\right\rangle_{A}, \\
\psi_{J_{\mathrm{f}} M_{\mathrm{f}}}(A+2)=\sum_{N^{\prime} v^{\prime} \eta^{\prime}} B_{N^{\prime} v^{\prime} \eta^{\prime}}^{J_{\mathrm{f}}}\left|N^{\prime} v^{\prime} \eta^{\prime} J_{\mathrm{f}} M_{\mathrm{f}}\right\rangle_{A+2} .
\end{gathered}
$$

Here $|N v \eta J M\rangle$ represents a many-phonon state built from a single collective quadrupole-type phonon $(|1102 M\rangle)$. The meaning of the quantum numbers is as follows: $N$ gives the number of phonons; $v$ gives the seniority of the state; $J M$ gives the total angular momentum and its $z$-component; $\eta$ is an additional quantum number needed to completely specify the state.

The coefficients $B_{N v \eta}^{J}$ are obtained by diagonalizing a model Hamiltonian according to the prescription given by the boson expansion method ${ }^{6}$ ).

The one-phonon state $|1102 M\rangle$ may be conveniently written in the language of second quantization by use of the creation (destruction) operators $\alpha_{2 M}^{+}\left(\alpha_{2 M}\right)$ which satisfy:

$$
\begin{gathered}
\alpha_{2 M}^{+}|0\rangle \equiv|1102 M\rangle, \\
\alpha_{2 M}|0\rangle=0, \\
{\left[\alpha_{2 M}, \alpha_{2 M^{\prime}}^{+}\right]=\delta_{M^{\prime}} .}
\end{gathered}
$$

The operator $\alpha_{2 M}^{+}$may in turn be expressed in terms of the operators $A_{2 M}^{+}$defined in eq. (3.3a) of ref. ${ }^{6}$ ) [see also discussion on p. 238 of ref. $\left.{ }^{6}\right)$ ] by use of the canonical transformation:

$$
\alpha_{2 M}^{+}=\frac{1}{2}\left(Z+Z^{-1}\right) A_{2 M}^{+}-\frac{1}{2}\left(Z-Z^{-1}\right) A_{2 M},
$$

where the parameter $Z$ is obtained by imposing the condition that the coefficient of $\left[\alpha_{2}^{+} \alpha_{2}^{+}\right]_{0}+\left[\alpha_{2} \alpha_{2}\right]_{0}$ vanishes from our model Hamiltonian ${ }^{7}$ ).

+ Although in (1) we have in mind a stripping process, our discussion may be applied to pickup reactions as well, provided we replace $\phi$ by $\phi^{*}$ and interchange $A \leftrightarrow A+2$. 
The vacuum of the $\alpha$-operators may be expanded in powers of these $A$-operators acting on the BCS ground state, i.e.

$$
|0\rangle=\left(C_{1}+C_{2}\left[A_{2}^{+} A_{2}^{+}\right]_{0}+\ldots\right)|\mathrm{BCS}\rangle .
$$

The coefficients $C_{i}$ in (5) arc casily obtained as solutions of (3b) and using the fact that $A_{2 M}|\mathrm{BCS}\rangle=0$.

In table $1 \mathrm{~A}$ we have tabulated for the case of the samarium isotopes the values of $C_{1}, C_{2}$ and $Z$.

It is essential for the evaluation of the overlap in eq. (1) to be able to express the target and residual nucleus wave function in terms of a common basis. We achieve this by exploiting the slow dependence with mass number of the parameter $Z$, the coefficient $C_{i}$ and the operator $A_{2 M}^{+}$.

Indeed as shown in table $1 B$ the overlaps ${ }_{A}\langle 1102 M \mid 1102 M\rangle_{A+2}$ are always larger than 0.9. We calculate these overlaps by first evaluating the overlaps ${ }_{A}\langle\mathrm{BCS}| A_{2 M} A_{2 M}^{+}$ $|\mathrm{BCS}\rangle_{A+2}$ and then using eqs. (4) and (5) with the corresponding values from table 1A. The overlaps ${ }_{A}\left\langle\mathrm{BCS}\left|A_{2 M} A_{2 M}^{+}\right| \mathrm{BCS}\right\rangle_{A+2}$ are in turn easily obtained by expressing $A_{2 M}^{+}|\mathrm{BCS}\rangle_{A}$ and $A_{2 M}^{+}|\mathrm{BCS}\rangle_{A+2}$ in terms of the same truncated spherical shell model basis, following ref. ${ }^{6}$ ).

TABLE $1 \mathrm{~A}$

The values of $Z, C_{1}$ and $C_{2}$ for the samarium isotopes

\begin{tabular}{lllll}
\hline Isotope & ${ }^{148} \mathrm{Sm}$ & ${ }^{150} \mathrm{Sm}$ & ${ }^{152} \mathrm{Sm}$ & ${ }^{154} \mathrm{Sm}$ \\
\hline$Z$ & 1.09 & 1.13 & 1.19 & 1.24 \\
$C_{1}$ & 0.989 & 0.979 & 0.962 & 0.946 \\
$C_{2}$ & 0.143 & 0.199 & 0.272 & 0.324 \\
\hline
\end{tabular}

TABLE 1B

The overlaps ${ }_{A}\left\langle\mathrm{BSC}\left|A_{2 M} A_{2 M}^{+}\right| \mathrm{BCS}\right\rangle_{A+2}$ and ${ }_{A}\langle 1102 M \mid 1102 M\rangle_{A+2}$

\begin{tabular}{llll}
\hline & $A=148$ & $A=150$ & $A=152$ \\
\hline${ }_{A}\left\langle\mathrm{BCS}\left|A_{2 M} A_{2 M}^{+}\right| \mathrm{BCS}\right\rangle_{A+2}$ & 0.9884 & 0.9953 & 0.9865 \\
${ }_{A}\langle 1102 M \mid 1102 M\rangle_{A+2}$ & 0.9652 & 0.9470 & 0.9100 \\
\hline
\end{tabular}

It is therefore possible to express the wave function of both target and residual nucleus in terms of the same many-phonon basis, i.e., we will replace the index $A+2$ in the right had side of eq. (2b) by $A$. (Since from now on we will refer everything to the many-phonon basis of the target nucleus, we will drop the index $A$ altogether.) We point out here that this does not mean that we assume the wave functions for the $\mathrm{Sm}$ isotopes to be $A$-independent; it rather means that the basis in which we expand the $\mathrm{Sm}$ wave functions is $A$-independent. The $A$-dependence of the wave function appears then only through the coefficients $B_{N_{v n}}^{J}$ of (2). 
Since our present boson expansion code BOSEXP $^{7}$ ) allows us to calculate only wave functions for the low-lying collective states of doubly even nuclei, we will restrict our study to the transfer of two identical particles only.

\subsection{THE FACTOR $\phi_{\gamma L S J T M_{T}}\left(\boldsymbol{r}_{1}, \boldsymbol{r}_{2}\right)$}

In order to evaluate the overlap (1), it is necessary to express $\phi_{y L S J T M_{T}}\left(\boldsymbol{r}_{1}, \boldsymbol{r}_{2}\right)$ in terms of boson creation and destruction operators which can act directly on the manyphonon basis $|N v \eta J M\rangle$, i.e., in terms of the operators defined in eq. (3). In order to accomplish this we generalize the procedure which was employed elsewhere ${ }^{6}$ ) to write the Hamiltonian in terms of boson operators. This generalization is needed since we now deal with an operator which is necessarily non-hermitian and in general a spherical tensor of rank greater than zero.

We rewrite $\phi$ as $^{\dagger}$

$\phi_{\gamma L S J M}\left(\boldsymbol{r}_{1}, \boldsymbol{r}_{2}\right)=\sum_{\substack{j_{1}=l_{1} \pm \frac{1}{3} \\ j_{2}=l_{2} \pm \frac{3}{2}}} \hat{L} \hat{j}_{1} \hat{j}_{2}\left\{\begin{array}{ccc}L & l_{1} & l_{2} \\ S & \frac{1}{2} & \frac{1}{2} \\ J & j_{1} & j_{2}\end{array}\right\} \Phi_{\gamma j_{1} j_{2} J M}\left(\boldsymbol{r}_{1}, \boldsymbol{r}_{2}\right)$,

where $\boldsymbol{r}_{1}$ and $\boldsymbol{r}_{2}$ are the coordinates of the two transferred nucleons referred to the c.m. of the target. We introduce the notation $\hat{A}=(2 A+1)^{\frac{1}{2}}$ and $D_{j_{1} j_{2}}=\left(1+\delta_{j_{1} j_{2}}\right)^{\frac{1}{2}}$, while $\gamma \equiv\left(n_{1}, l_{1} ; n_{2}, l_{2}\right)$ where $n_{i} l_{i}$ stand for the principal and orbital angular momentum quantum numbers of the orbit occupied by the transferred nucleon $i$.

Furthermore:

$\Phi_{\gamma j_{1} j_{2} J M}\left(\boldsymbol{r}_{1}, \boldsymbol{r}_{2}\right)=A\left[\left[\varphi_{n_{1} l_{1}}\left(\boldsymbol{r}_{1}\right) \chi_{\frac{1}{2}}(1)\right]_{j_{1}}\left[\varphi_{n_{2} l_{2}}\left(\boldsymbol{r}_{2}\right) \chi_{\frac{1}{2}}(2)\right]_{j_{2}}\right]_{J M} \equiv\left[a_{j_{1}}^{+} a_{j_{2}}^{+}\right]_{J M} D_{j_{1} j_{2}}^{-1}$.

We have chosen harmonic oscillator eigenstates for the $\varphi_{n_{2} l_{2}}\left(\boldsymbol{r}_{2}\right)$. The $\chi_{\frac{1}{2}}(i)$ stands for the spin part of the wave function of particle $i$. Finally we have introduced the notation of second quantization which we will use throughout.

We now introduce the quasiparticle $d_{j m}^{+}$by performing a Bogoliubov transformation:

$$
a_{j m}^{+}=u_{j} d_{j m}^{+}-v_{j} d_{j \tilde{m}},
$$

where $d_{j \tilde{m}}=(-)^{j-m} d_{j-m}$. We then obtain for $\phi$ :

$$
\begin{aligned}
\phi_{\gamma L S J M}=-\sum_{j_{1}} \hat{j}_{1} R_{j_{1} j_{1}} u_{j_{1}} v_{j_{1}} \delta_{J 0} \delta_{j_{1} j_{2}} & \delta_{l_{1} t_{2}} \frac{1}{2}\left(1+(-)^{L+S}\right) \\
& +\frac{1}{2} \sum_{j_{1} j_{2}} S R_{j_{1_{1}} j_{2}}\left[u_{j_{1}} u_{j_{2}} B_{j_{j_{2}} J M}^{+}-v_{j_{1}} v_{j_{2}} B_{j_{1} j_{2} J \tilde{M}}\right] \\
& -\frac{1}{2} \sum_{j_{1} j_{2}} S R_{j_{1_{1}} j_{2}}\left[u_{j_{1}} v_{j_{2}} C_{j_{j_{2}} J M}^{+}+u_{j_{2}} v_{j_{1}} C_{j_{1_{1}} J \tilde{M}}\right]
\end{aligned}
$$

+ From here on we will always assume $T=1$. Furthermore, the dependence of $\phi$ on $M_{T}$ is trivial so it will be ignored. 
where

$$
\begin{gathered}
R_{j_{1} j_{2}}=D_{j_{1} j_{2}}^{-1} L \hat{S} \hat{j_{1}} \hat{j}_{2}\left\{\begin{array}{ccc}
L & l_{1} & l_{2} \\
S & \frac{1}{2} & \frac{1}{2} \\
J & j_{1} & j_{2}
\end{array}\right\} \\
S=\left(1+(-)^{L+S+l_{1}+l_{2}}\right) \\
B_{j_{1} j_{2} J M}^{+}=\left[d_{j_{1}}^{+} d_{j_{2}}^{+}\right]_{J M}, \\
C_{j_{1} j_{2} J M}^{+}=\left[d_{j_{1}}^{+} d_{j_{2}}\right]_{J M}
\end{gathered}
$$

At this stage we extract the collective mode by performing a Tamm-Dancoff approximation (TDA) on the two-quasiparticle states by using a $Q \cdot Q$ type of residual interaction of the same strength as the one which was used in obtaining the wave functions of the target nucleus. This amounts to performing an orthogonal transformation on the two-quasiparticle states of the form:

$$
B_{j_{1} j_{2} J M}^{+}=\sum_{\alpha} D_{j_{1} j_{2}} \psi_{J_{1} j_{2} J}^{(\alpha)} B_{\alpha J M}^{+} \quad\left(j_{1} \leqq j_{2}\right)
$$

Here we reserve $\alpha=0$ to denote the collective branch.

We then obtain for $\phi$ :

$$
\begin{aligned}
\phi_{\gamma L S J M}=-\sqrt{\frac{1}{2}} \frac{1}{2}(1+(- & )^{L+S}\right) \sum_{j_{1}} \hat{j}_{1}^{2} u_{j_{1}} v_{j_{1}} \hat{L} W\left(l_{1} L j_{1} \frac{1}{2} ; l_{1} \frac{1}{2}\right) \delta_{J 0} \delta_{j_{1} j_{2}} \delta_{l_{1} l_{2}} \\
& +\sum_{\alpha}\left(F_{\alpha J}^{u u} B_{\alpha J M}^{+}-F_{\alpha J}^{v v} B_{\alpha J \tilde{M}}\right)-\sum_{p}\left(F_{p}^{u v} C_{p J M}^{+}+F_{p}^{v u} C_{p J \tilde{M}}\right)
\end{aligned}
$$

where

$$
\begin{gathered}
F_{\alpha J}^{u u}=\sum_{j_{1} j_{2}} R_{j_{1} j_{2}} D_{j_{1} j_{2}} S u_{j_{1}} u_{j_{2}} \psi_{j_{1} j_{2} J}^{(\alpha)}, \\
F_{\alpha J}^{v v}=\sum_{j_{1} j_{2}} R_{j_{1} j_{2}} D_{j_{1} j_{2}} S v_{j_{1}} v_{j_{2}} \psi_{j_{1} j_{2} J}^{(\alpha)}, \\
F_{p}^{u v}=R_{j_{1} j_{2}} u_{j_{1}} v_{j_{2}} S \\
F_{p}^{v u}=R_{j_{1} j_{2}} u_{j_{2}} v_{j_{1}} S
\end{gathered}
$$

and $p$ stands for the pair $\left(j_{1}, j_{2}\right)$.

We proceed now to expand the fermion pair operators in terms of boson operators. We do this following the procedure introduced by Belyaev and Zelevinsky ${ }^{4}$ ).

The expansion takes the form ${ }^{6}$ ):

$$
\begin{gathered}
B_{\alpha J M}^{+}=x A_{a}^{+} \delta_{J 2}+\sum_{b c d} X_{3}(a b c d) A_{b}^{+} A_{c}^{+} A_{d}+\ldots \\
C_{p J M}^{+}=x_{0}+\sum_{a b} X_{2}(p, a b) A_{a}^{+} A_{b}+\ldots
\end{gathered}
$$


The boson operators $A_{i}$ satisfy:

$$
\left[A_{i}, A_{j}^{+}\right]=\delta_{i j} \text {. }
$$

Except for the coefficient $x$ all the other coefficients in the expansion are determined by requiring that the fermion pair operators satisfy the appropriate commutation relations. We assign to $x$ the value used in the wave function of the target nucleus in the case of stripping, while for pickup we use the value used for the wave function of the residual nucleus. Furthermore, since eventually we will have to take matrix elements of these boson operators between states of the form (2) only those operators which correspond to $\alpha=0$ will survive. Therefore, we retain only the component which relates to the collective branch. Upon inserting (14) in (12) and rearranging, we obtain:

$$
\phi_{\gamma L S J M}=\sum_{m n i} g_{m n i}^{(A)} G_{m n i}^{(A)},
$$

where the operators $G_{m n i}^{(A)}$ with $m \geqq n$ are defined by:

$$
\begin{array}{ll}
G_{0}^{(A)}=1, & G_{21 N}^{(A)}=\sqrt{5} A_{2 M}^{+}\left[A_{2}^{+} A_{2}\right]_{0}, \\
G_{21 V}^{(A)}=\sqrt{5}\left[A_{2}^{+} A_{2}^{+}\right]_{0} A_{2 \tilde{M}}, & G_{30 V}^{(A)}=\sqrt{5} A_{2 M}^{+}\left[A_{2}^{+} A_{2}^{+}\right]_{0}, \\
G_{20}^{(A)}=\left[A_{2}^{+} A_{2}^{+}\right]_{J M}, & G_{11}^{(A)}=\left[A_{2}^{+} A_{2}\right]_{J M}, \\
G_{30 K}^{(A)}=\left[\left[A_{2}^{+} A_{2}^{+}\right]_{K} A_{2}^{+}\right]_{J M}, & G_{10}^{(A)}=A_{2 M}^{+}, \\
G_{21 K}^{(A)}=\left[A_{2}^{+}\left[A_{2}^{+} A_{2}\right]_{K}\right]_{J M} . &
\end{array}
$$

The corresponding operators with $m \leqq n$ are defined by:

$$
G_{m n i}^{(A)}=G_{n m i}^{(A)+} .
$$

The coefficients $g_{m n i}^{(A)}$ in (16) are given by:

where

$$
\begin{aligned}
& g_{0}^{(A)}=\sqrt{\frac{1}{2}}\left(x^{2}-2\right) \sum_{j_{1}} \hat{j}_{1}^{2} u_{j_{1}} v_{j_{1}} \hat{L} W\left(l_{1} L j_{1} \frac{1}{2} ; l_{1} \frac{1}{2}\right) \frac{1}{2}\left(1+(-)^{L+S}\right), \\
& g_{10}^{(A)}=x F_{02}^{u u}, \quad g_{01}^{(A)}=-x F_{02}^{v v}, \\
& g_{21 N}^{(A)}=2 r F_{02}^{u u}, \quad g_{12 N}^{(A)}=-2 r F_{02}^{v v}, \\
& g_{11}^{(A)}=-\sum_{p}\left(F_{p}^{u v}+(-)^{J} F_{p}^{v u}\right) \hat{P}_{0,0}^{(p, J)} \\
& g_{21 K}^{(A)}=\frac{s \hat{K}}{\hat{J}}(-)^{J} \sum_{\alpha} F_{\alpha J}^{u u} f_{K}(\alpha J) \\
& g_{12 K}^{(A)}=-\frac{s \hat{K}}{\hat{J}} \sum_{\alpha} F_{\alpha J}^{v v} f_{K}(\alpha J) \\
& \quad r=\frac{1}{6}\left(2 \sqrt{x^{2}-1}+\sqrt{x^{2}+2}-3 x\right) \\
& \quad s=\frac{1}{6}\left(\sqrt{x^{2}-1}-\sqrt{x^{2}+2}\right),
\end{aligned}
$$


while

$$
\begin{gathered}
f_{K}(\alpha J)=(-)^{J} \sum_{j_{1} j_{2}} \hat{P}_{\alpha J, 0}^{\left(J_{1} j_{2} K\right)} \hat{P}_{0,0}^{\left(j_{1} j_{2} K\right)} \\
\hat{P}_{\alpha, j, 0}^{\left(j_{1} j_{2} K\right)}=\sqrt{5} \hat{J} \sum_{j} \psi_{j_{j 2} J}^{(\alpha)} \psi_{j_{1} j_{2}}^{(\alpha)} D_{j_{1 j} j} D_{j_{1 j}} W\left(j_{2} j_{1} 2 J ; K j\right)
\end{gathered}
$$

As a last step we now need to perform a final canonical transformation within the A-boson space given by

$$
\alpha_{2 M}^{+}=\frac{1}{2}\left(Z+Z^{-1}\right) A_{2 M}^{+}-\frac{1}{2}\left(Z-Z^{-1}\right) A_{2 \tilde{M}} .
$$

Again we chose for $Z$ the value obtained for the target wave function in the case of stripping. Explicitly then our operators in the $A$-representation may be expressed in terms of the corresponding operators in the $\alpha$-representation in the following way:

$$
\begin{aligned}
G_{10}^{(A)}= & \frac{1}{2} A G_{10}^{(\alpha)}+\frac{1}{2} a G_{01}^{(\alpha)}, \\
G_{11}^{(A)}= & \frac{1}{4} b\left(G_{20}^{(\alpha)}+G_{02}^{(\alpha)}\right)+\frac{1}{4}\left(A^{2}+(-)^{J} a^{2}\right) G_{11}^{(\alpha)}, \\
G_{21 N}^{(A)}= & \frac{1}{8}(3 C-3 A) G_{10}^{(\alpha)}+\frac{1}{8}(3 c-5 a) G_{01}^{(\alpha)}+\frac{1}{4}(C+A) G_{21 N}^{(\alpha)}+\frac{1}{4}(c-a) G_{12 N}^{(\alpha)}+\frac{1}{8}(C-A) G_{1 V}^{(\alpha)} \\
& \quad+\frac{1}{8}(c+a) G_{12 V}^{(\alpha)}+\frac{1}{8}(c+a) G_{30 V}^{(\alpha)}+\frac{1}{8}(C-A) G_{03 V}^{(\alpha)}, \quad(24) \\
G_{21 K}^{(A)}= & \frac{1}{8}(C-A) G_{10}^{(\alpha)}+\frac{1}{8}(c-3 a) G_{01}^{(\alpha)}+\frac{1}{8}(-)^{J}(c+a) G_{30 K}^{(\alpha)}+\frac{1}{8}(-)^{J}(C-A) G_{03 K}^{(\alpha)} \\
+ & \sum_{l}\left(\frac{1}{8}(C-A) \hat{K} \hat{l}(-)^{J} W \quad(J 222 ; K l)+\delta_{K l}\left[\frac{1}{8}(C+c+3 A+a)+\frac{1}{8}(-)^{K}(C-A)\right]\right) G_{21 l}^{(\alpha)} \\
& +\sum_{l}\left(\frac{1}{8}(c+a) \hat{K} \hat{l} W(J 222 ; K l)+\frac{1}{8} \delta_{K l}(c-3 a)\right) G_{12 l}^{(\alpha)},
\end{aligned}
$$

with

$$
\begin{array}{ll}
A=Z+Z^{-1}, & a=Z-Z^{-1}, \\
B=Z^{2}+Z^{-2}, & b=Z^{2}-Z^{-2}, \\
C=Z^{3}+Z^{-3}, & c=Z^{3}-Z^{3} .
\end{array}
$$

We have therefore succeeded in expressing $\phi$ as a linear combination of operators which can act directly on the many-phonon basis, i.e.,

and so finally

$$
\phi_{\gamma L S . M}=\sum_{m n i} g_{m n i}^{(\alpha)} G_{m n i}^{(\alpha)}
$$

$\beta_{\gamma L S J M}^{\left(J_{1}, J_{\mathrm{f}}\right)}=\sum_{N v \eta} \sum_{N^{\prime} v^{\prime} \eta^{\prime}} \sum_{m+n i} B_{N^{\prime} v \eta}^{J_{i}} B_{N^{\prime} v^{\prime} \eta^{\prime}}^{J_{\mathrm{f}}} g_{m n i}^{(\alpha)}\left\langle N^{\prime} v^{\prime} \eta^{\prime} J_{\mathrm{f}}\left|G_{m n i}^{(\alpha)}\right| N v \eta J_{\mathrm{i}}\right\rangle$

In order to evaluate (27) we only need the matrix element of the $G$-operators between many-phonon states. We have obtained these matrix elements by generalizing the procedure described in ref. ${ }^{8}$ ) to include tensor operators of rank greater than zero. 


\section{Calculations}

In this section we apply the formulation of the previous section to obtain spectroscopic factors for two-nucleon transfer reactions for the $\mathrm{Sm}$ isotopes. In fig. 1 we have compared the experimental spectra with those obtained by performing a sixthorder boson expansion calculation for the isotopes ${ }^{148,150,152,154} \mathrm{Sm}$. The details

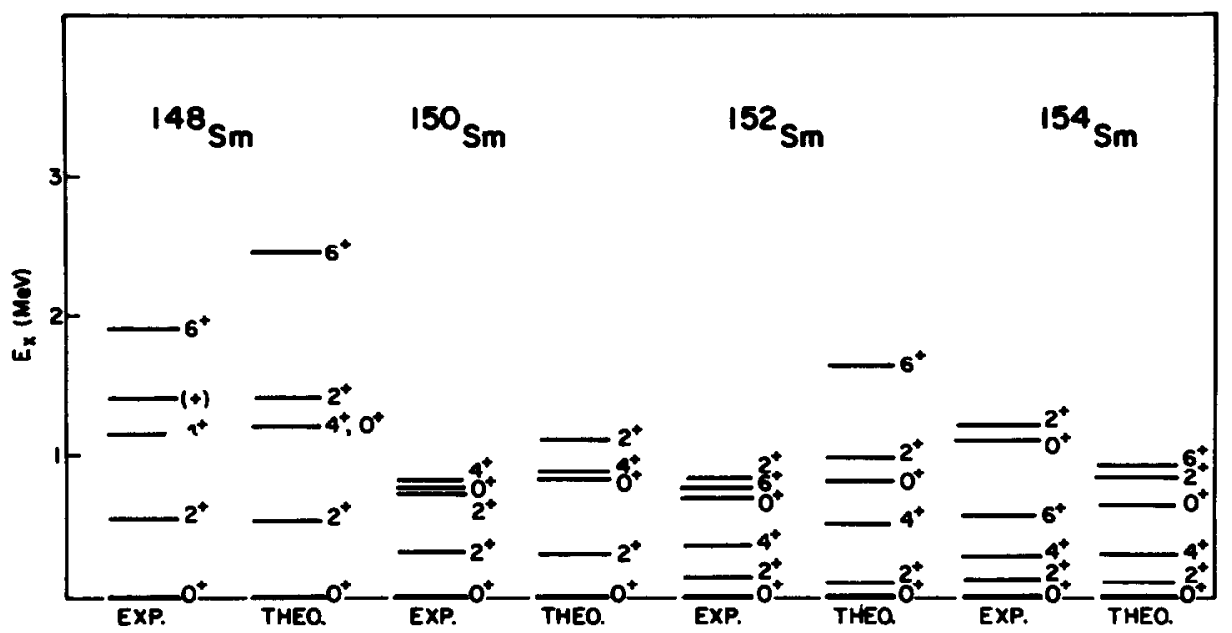

Fig. 1. Comparison of energy levels obtained with a boson expansion calculation with experimental values from ref. ${ }^{16}$ ), for the isotopes ${ }^{148,150,152,154} \mathrm{Sm}$.

of these calculations have been reported elsewhere ${ }^{7}$ ). The theoretically predicted spectra do account for the transition from a vibrational to a rotational spectrum. This is further corroborated if we use the boson expansion wave functions to calculate the quadrupole moment of the first $2^{+}$state as well as electromagnetic transition strengths for members of the ground-state band. The results are given in table 2 . The effective charge $\left(e_{\mathrm{eff}}\right)$ is defined as $e_{\text {proton }}=1.0+e_{\text {eff }}$. The good agreement with experiment should be noted.

The boson expansion wave functions used are given explicitly in table 3 . These were obtained by using our boson expansion code BOSEXP. It is important to point out that the character of these wave functions is a sensitive function of the expansion parameter $x$. We normally determine $x$ by using an automatic search option provided in BOSEXP which adjusts $x$ so that the energy of the $2^{+}$state is approximately reproduced ${ }^{7}$ ). In table 3 we have used a shorthand notation for the many boson states $|N v \gamma J M\rangle$ in which we drop the quantum numbers $\gamma J M$, since $J$ and $M$ are implied by the left hand side of the state vector equation. (The $\gamma$-multiplicity when needed is to be understood through the appearance of consecutive repetitions of the pair $N v$.) We have also ignored components of magnitude less than $10^{-3}$; this allows us to truncate the expansion so that $N \leqq 8$. In this table we also include the value used for $x$. 

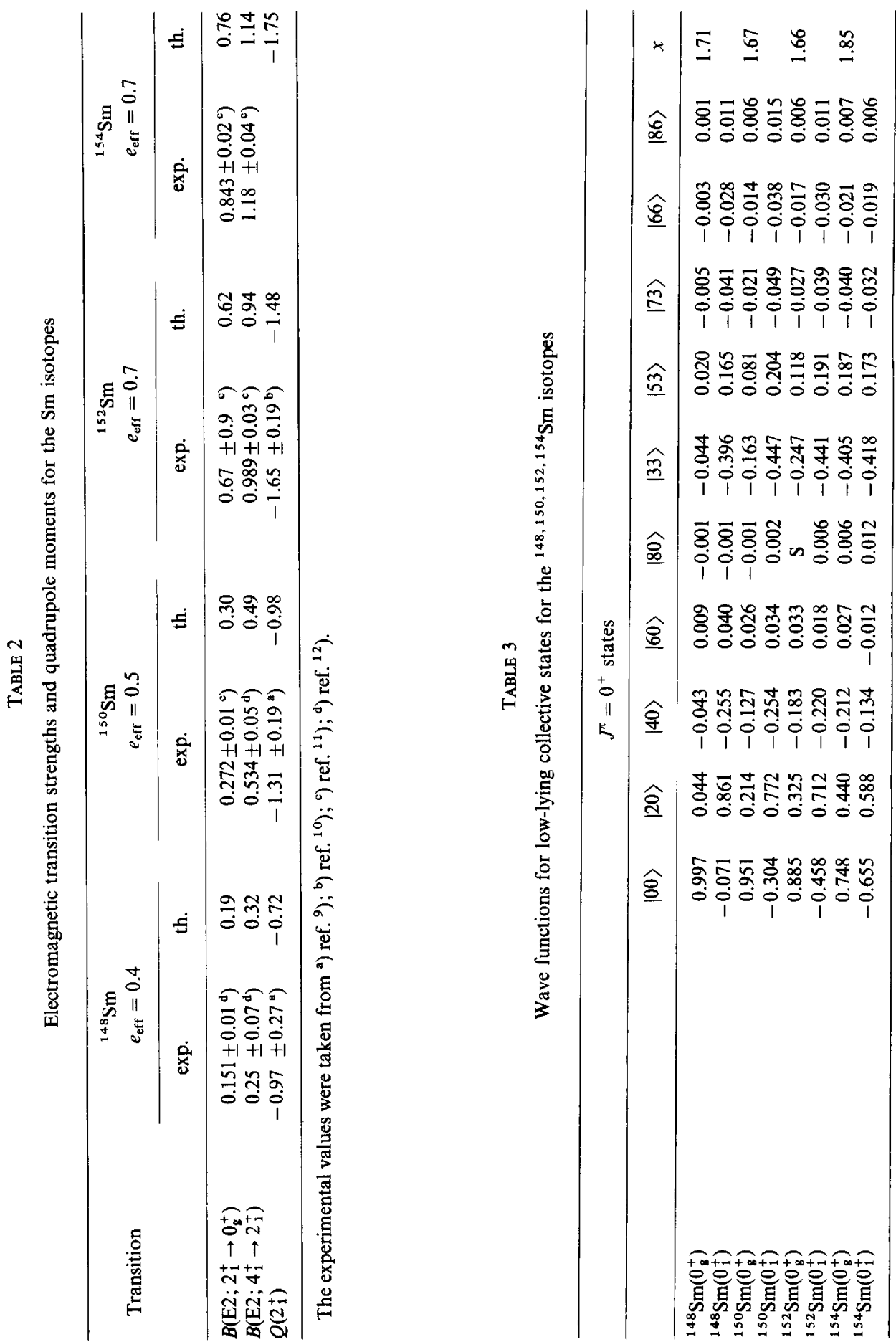





Using the wave functions given in table 3 and the formalism presented in the previous section, we have calculated spectroscopic factors for two-neutron stripping reactions on ${ }^{148,150,152} \mathrm{Sm}$. The results are presented in table 4 . The table can also be used for two-neutron pickup provided we interchange $J_{\mathrm{i}} \leftrightarrow J_{\mathrm{f}}$ and multiply each entry by $\sqrt{2 J_{\mathrm{f}+1} / 2 J_{\mathrm{i}+1}}$. Instead of tabulating the spectroscopic amplitude $\beta_{\gamma L S J T}$ as given by (1), we have chosen to tabulate $\beta_{\gamma j_{1} j_{2} J T}$ defined by:

$$
\beta_{\gamma j_{1} j_{2} J}^{\left(J_{1}, J_{\mathrm{f}}\right)}=5 \sum_{L S} \hat{L} \hat{S} \hat{j}_{1} \hat{j_{2}}\left\{\begin{array}{lll}
L & l_{1} & l_{2} \\
S & \frac{1}{2} & \frac{1}{2} \\
J & j_{1} & j_{2}
\end{array}\right\} \beta_{\gamma L S J}^{\left(J_{1}, J_{\mathrm{f}}\right)}
$$

this can then be used directly in existing DWBA codes such as DWUCK ${ }^{15}$ ). All non-zero quantities which are smaller than $10^{-3}$ have been tabulated as $\mathrm{S}$.

By inspection of the tables, we may arrive at the following conclusions:

(i) As we go to heavier isotopes of $\mathrm{Sm}$, we see a gradual shift of strength to higherlying levels, which is expected due to partial filling of low-lying orbits.

(ii) A relative concentration of strength for $J=2$ when several transfer $J$-values are allowed. This is due to the fact that we are expanding our states in terms of a basic quadrupole-type phonon. As we shall see below, there is an indication that it might be desirable to include a monopole type of excitation as well.

(iii) The apparent importance of higher-lying levels such as $1 \frac{13}{2}$ is largely offset when we perform the transformation to c.m. and relative coordinates since the transformation coefficient (Moshinsky coefficient) favors small spins.

We expect the $0_{\mathbf{g}}^{+} \rightarrow 0_{\mathbf{g}}^{+}$transitions to be dominated by direct processes. These transitions should therefore provide a good test of the reliability of the predicted spectroscopic amplitudes, particularly in their variation with mass number through the transition region from $A=148$ to $A=152$. For this purpose we have used the twoparticle transfer option of DWUCK. The optical potential parameters for the proton and triton were obtained from refs. ${ }^{13,14}$ ) respectively.

The theoretical cross section was then obtained by multiplying the DWUCK cross section by the following factors ${ }^{15}$ ):

$$
\sigma_{\text {theor }}=\frac{D_{0}^{2}}{25}\left(\frac{\pi \Delta^{2}}{2}\right)^{\frac{3}{2}}\left\langle T_{\mathrm{f}} M_{\mathrm{f}} 11 \mid T_{\mathrm{i}} M_{\mathrm{i}}\right\rangle^{2}\left(\begin{array}{c}
n+2 \\
2
\end{array}\right) \sigma_{\text {DwUCK }}
$$

where we have assumed the standard value ${ }^{15}$ ) of $D_{0}^{2}=22 \mathrm{MeV} \cdot \mathrm{fm}^{3}$, while

$$
\left(\frac{1}{2} \pi \Delta^{2}\right)^{\frac{3}{2}}=9.72 \mathrm{fm}^{3} .
$$

The $T_{\mathrm{i}}$ and $T_{\mathrm{f}}$ stand for the target and residual nucleus isospin, respectively. The binomial coefficient is a statistical factor where $n$ is the number of valence neutrons.

In fig. 2 we have compared the calculated cross section with experimental data obtained from ref. ${ }^{17}$ ). The plotted cross section is related to the theoretical cross sec- 


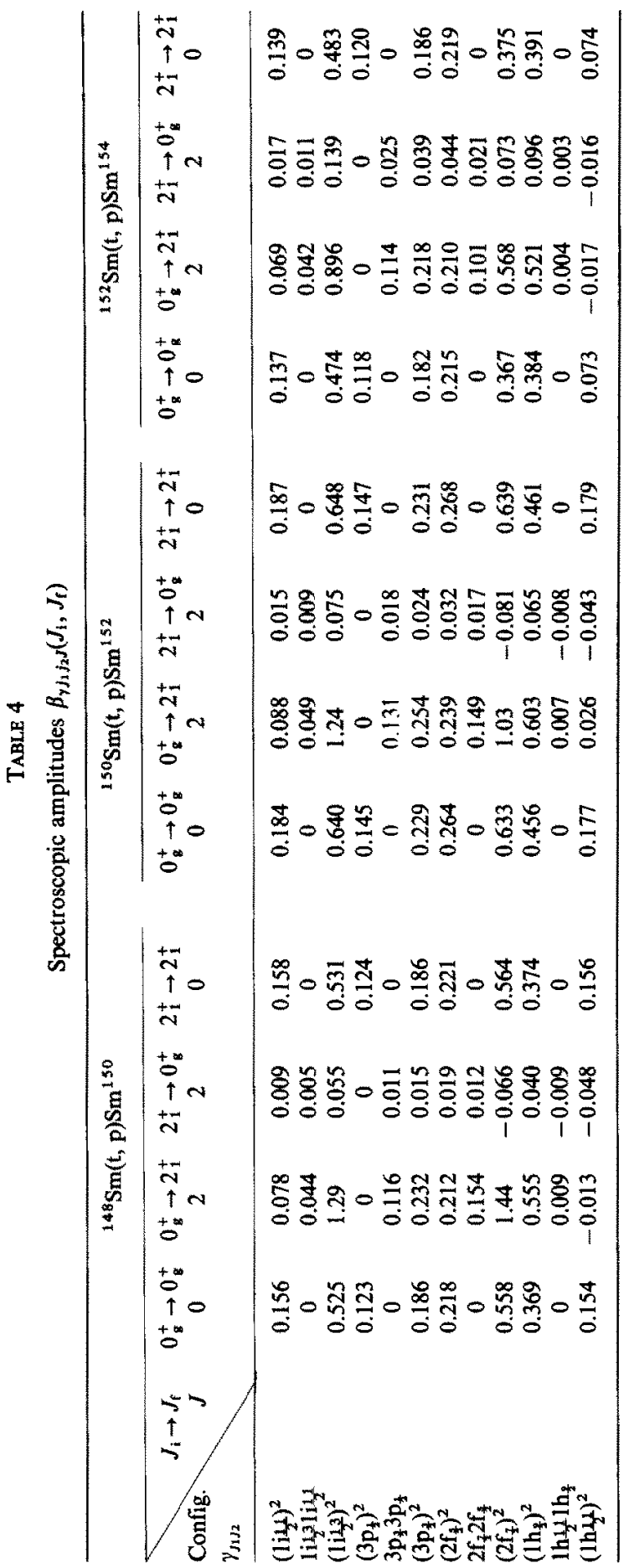



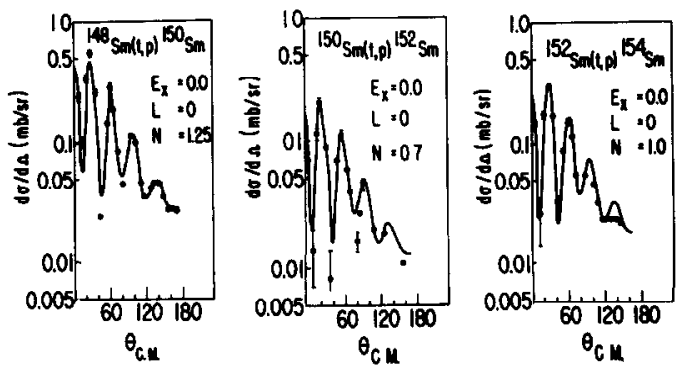

Fig. 2. Calculated and experimental cross sections for $(t, p)$ reactions on the ${ }^{148,150,152} \mathrm{Sm}$ targets. The mormalization constant $N$ indicates the goodness of the prediction of the absolute value of the transfer amplitude.

tion by:

$$
\sigma_{\text {plot }}=N^{2} \sigma_{\text {theory }},
$$

where the normalization constant $N$ gives a measure of how well the absolute cross sections are predicted. As shown in fig. 2, we are indeed capable of predicting them rather well for transitions leading to the ground state. This is indicated by the fact that we used for $N$ a value close to 1 . For the transitions within the deformed region $\left({ }^{152} \mathrm{Sm}(\mathrm{t}, \mathrm{p}){ }^{154} \mathrm{Sm}\right)$, we use $N=1.0$. The excellent agreement in this region is probably due to the fact that the coupling to non-collective branches is unimportant. This is also in agreement with the accurate prediction of electromagnetic transition strengths provided by the boson expansion method in this region. In the spherical region $\left({ }^{148} \mathrm{Sm}(\mathrm{t}, \mathrm{p}){ }^{150} \mathrm{Sm}\right)$ we also account nicely for the transition to the ground state $(N=1.25)$. This is indeed remarkable if we recall that our calculation is completely free of adjustable parameters.

For transitions to excited states, it is known ${ }^{18}$ ) that inelastic effects in the reaction channels are important. Furthermore, it is known ${ }^{19}$ ) that in the rare-earth region the ratio $\sigma\left(4_{1}^{+}\right) / \sigma\left(2_{1}^{+}\right)$in $(\mathrm{p}, \mathrm{t})$ and $(\mathrm{t}, \mathrm{p})$ reactions is of the order of 0.3 . An inspection of the table of spectroscopic amplitudes shows that the direct process $\mathrm{O}_{\mathrm{g}}^{+} \rightarrow 4_{1}^{+}$is severely inhibited, while two-step processes could be quite strong. We therefore believe that excited phonon states can only be properly described by a coupled channels approach. In table 4 we have also included spectroscopic amplitudes which lead to the onephonon state, and that could be used in a coupled channels calculation. We defer this question to a future study.

\section{Summary}

We have presented in sect. 2 the essentials of the formalism which is needed to calculate spectroscopic amplitudes for two-particle transfer reactions with wave functions provided by a boson expansion calculation. In sect. 3 we have applied this for- 
malism to the isotopes ${ }^{148,150,152,154} \mathrm{Sm}$ and obtained extensive tables of spectroscopic amplitudes for various possible transitions involving low-lying collective states. We emphasize that our calculations are completely parameter-free. Therefore, we believe we have developed a technique which allows calculations of reliable spectroscopic amplitudes for two-nucleon transfer reactions regardless of the target or residual nucleus deformation. Using these spectroscopic amplitudes we have then evaluated the cross section for $(t, p)$ reactions on ${ }^{148,150,152} \mathrm{Sm}$. We have obtained excellent results for transitions leading to the ground state and one phonon state in the residual nucleus. These results confirm our expectation that the boson expansion wave functions do indeed offer a good description of low-lying collective states.

The author gratefully acknowledges Professor K. T. Hecht for many enlightening discussions and useful suggestions during the course of this work.

\section{References}

1) N. K. Glendenning, Phys. Rev. 137 (1965) B102

2) S. Yoshida, Nucl. Phys. 33 (1961) 685

3) G. Satchler, Ann. of Phys. 3 (1958) 275

4) S. Belyaev and V. Zelevinsky, Nucl. Phys. 39 (1962) 582

5) B. Sфrensen, Nucl. Phys. A97 (1967) 1; A119 (1968) 65; A142 (1970) 392; A142 (1970) 411

6) T. Kishimoto and T. Tamura, Nucl. Phys. A192 (1972) 246

7) D. Braunschweig, Doctoral Dissertation, Univ. of Texas, 1973

8) T. Kishimoto and T. Tamura, Nucl. Phys. A163 (1971) 100

9) D. Vline et al., paper V5, Osaka Conf. (1972)

10) G. Casper et al., Proc. Conf. on heavy ions, Heidelberg, 1969

11) F. Stephens, R. Diamond and J. de Boer, Phys. Rev. Lett. 27 (1971) 1151

12) R. Diamond and F. Stephens, Phys. Rev. C3 (1972) 334

13) F. Becchetti and G. Greenlees, Phys. Rev. 182 (1969) 1190

14) F. Becchetti, W. Makofske and G. Greenlees, Nucl. Phys. A190 (1972) 437

15) H. Baer et al., Ann. of Phys. 76 (1972) 437

16) C. Lederer, J. Hollander and I. Perlman, in Table of isotopes (Wiley, New York, 1968)

17) J. Bjerregaard, O. Hansen, O. Nathan and S. Hinds, Nucl. Phys. 86 (1966) 145

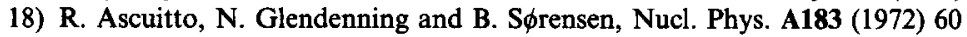

19) R. Casten and J. Garrett, Phys. Lett. 47B (1973) 436 